
Revue canadienne de chimie

\title{
Biological Study of Metal-organic Frameworks towards Human Ovarian Cancer Cell Lines
}

\begin{tabular}{|r|l|}
\hline Journal: & Canadian Journal of Chemistry \\
\hline Manuscript ID & Cjc-2015-0370 \\
\hline Manuscript Type: & Article \\
\hline Complete List of Authors: & $\begin{array}{r}\text { Wei, Zhangwen; Texas A\&M University, Department of Chemistry } \\
\text { Magnati, Madhuri ; Texas A\&M University-Kingsville, Department of } \\
\text { Chemistry } \\
\text { Martinez, Baldemar; Texas A\&M University-Kingsville, Department of } \\
\text { Chemistry } \\
\text { Vangara, Kiran ; Texas A\&M Rangel College of Pharmacy } \\
\text { Palakurthi, Srinath; Texas A\&M Rangel College of Pharmacy } \\
\text { Luo, Zhiping; Fayetteville State University, Department of Chemistry and } \\
\text { Physics } \\
\text { Bashir, Sajid; Chemical Biology Research Group (CBRG), } \\
\text { Zhou, Hong-Cai; Texas A\&M University, Department of Chemistry } \\
\text { Liu, Jingbo; Texas A\&M University, Department of Chemistry }\end{array}$ \\
\hline Keyword: & $\begin{array}{l}\text { Polycordinated Network (PCN), Metal-organic framework (MOF), Retinal } \\
\text { pigment epithelium cells, nitric oxide, cancer, fluorescenc }\end{array}$ \\
\hline & \\
\hline \multicolumn{2}{|c|}{} \\
\hline
\end{tabular}




\section{Biological Study of Metal-organic Frameworks towards Human Ovarian Cancer Cell Lines}

Zhangwen Wei, ${ }^{a}$ Madhuri Magnati, ${ }^{b}$ Baldemar Marinez, ${ }^{b}$ Kiran Kumar Vangara, ${ }^{c}$ Srinath. Palakurthi, ${ }^{c}$ Zhiping Luo, ${ }^{d}$ Sajid Bashir, ${ }^{b}$ Hong-Cai. Zhou* ${ }^{a}$ and Jingbo Liu* ${ }^{a, b}$

a Department of Chemistry, Texas A\&M University College Station, TX 77843-3255 , USA. b Department of Chemistry, Texas A\&M University-Kingsville, TX 78363-8202, USAc Texas A\&M Rangel College of Pharmacy, 1010 W. Ave. B., Kingsville, TX 78363-8202, USA. d Department of Chemistry and Physics, Fayetteville State University, Fayetteville, NC 283014252, NC, USA.

*Corresponding author .. Fax: 01361593 3597; Tel: 01361593 2919; E-mail:

KFJLL00@tamuk.edu (LL); Tel: 013615934253 


\begin{abstract}
Solvothermal chemistry was employed to produce PCN-95 starting with pyrene forming 8connected Zr6 clusters conforming to isoreticular structure (P6/mmm). The secondary building unit was determined from polycrystals as having Zr6 center octahedral to and covering all triangular faces with eight $\mathrm{OH}$ and carboxylates as the framework. The PCN material was characterized by electron microscopy and evaluated from its cytotoxicity against Chinese hamster ovarian cells. The concentration at fifty-percent inhibition $\left(\mathrm{IC}_{50}\right)$ was in the low partsper-million and electron micrograph showed "darker region" around the deoxyribonucleic acid (DNA), indicative of DNA condensation leading to fragmentation. TO determine whether the observed biological effects were due to the generation of singlet oxygen or elicitation of specific death pathways; PCN with and without select inhibitors, reducing and oxidizing agents was employed against human, retinal pigment epithelium (RPE) in vitro and nitric oxide (NO) measured as a measure of cell health against controls cells (without any cofactors). The NO release was measured over $3 \mathrm{~h}$ time period and the kinetic plots compared and contrasted with known biochemical pathways. The plots were similar to methylene blue, a known singlet oxygen generator and between hydrogen peroxide and citrate but unlike cyanide or rotenone. Collectively the results support the hypothesis of singlet oxygen generation at the mitochondria and interaction with complex IV of the electron transport chain, facilitating the reduction of nitrite towards more NO. When the cell generates higher concentrations of NO, the apoptotic pathways are triggered resulting in cell death.
\end{abstract}

Keywords: Polycordinated Network (PCN), Metal-organic framework (MOF), Retinal pigment epithelium cells, nitric oxide, cancer, fluorescence, cytotoxicity 


\section{Introduction}

Metal-organic frameworks were produced and characterized, for chemical characteristics and biological activity against human ovarian cancer cells. To fully understand the therapeutic efficacy and mechanism of action, Chinese Hamster ovarian model cells were used, in four broad steps: 1) the materials synthesis; 2) the structural characterization; 3) properties measurement; and 4) the efficiency of the theranostics analysis.

Nanotheranostics based treatment requires precise control of size and functionality of the nanomaterial, that exhibit the "nanotechnology advantage" of large surface area to volume ratio, controlled size, hydrophobicity and surface charge according to intended application made them valuable carriers for therapeutic drugs and genes (Liu, 2012). ${ }^{1}$ Examples of drug-loaded scaffold systems include Genexol-PM (paclitaxel loaded micelles), abraxane (human serum albumin folded incorporation paclitaxel) and doxorubicin-liposomes. Theranostics is the dual application of diagnostics and therapy allowing for individualized pharmacogenomics, which are designed to minimise adverse drug reactions due to mismatched alleles and enzyme catalysis of drug to metabolites. The key features of the theranostics approach are (1) high drug loading, (2) high stability of the nanosystem-drug platform outside the target area, (3) drug release (low stability) inside the cancer cells, as well as (4) amplification of signal, typically as NIR fluorescence (for NIRF imaging) although this amplification may be tuned for magnetic resonance imagining, or positron emission tomography, depending on the nanoconstruct, and (5) low in vivo blood elimination kinetics for persistence and long-term effaicy. ${ }^{2}$ 
The nanocarrier platform can be liposomes ${ }^{3}$, micelles ${ }^{4}$, inorganic nanoparticles ${ }^{5}$, metal nanoparticles ${ }^{6}$, carbon nanotubes ${ }^{7}$, graphene ${ }^{8}$ or metal organic framework (MOFs) ${ }^{9}$. Each system has certain advantages and disadvantages; currently our approach was to use MOFs, due to the ability of high drug loading and high nanocarrier stability. MOFs have been studied intensively due to their permanent porosity and ultra-high internal surface areas. ${ }^{1}$ These crystalline inorganic-organic hybrids are composed of inorganic metal nodes and organic linkers. ${ }^{10-14}$ Tunability is achieved through changing the central metal element and synthesis of organic linkers from a large subset of potential scaffolds. Numerous MOFs have been designed and synthesized for application potentials in gas storage/separation, ${ }^{15-18}$ catalysis, ${ }^{19-21}$ sensing, 22-24 and drug delivery. ${ }^{25-27}$

The novel contributions of this research are: 1) preparation and characterization of zirconium (Zr) MOFs to introduce tetra-topic carboxylic groups; 2) generation of ultrahigh surface area (> $2000 \mathrm{~m}^{2} / \mathrm{g}$ ); and 3) evaluation of efficacy of $\mathrm{Zr}-\mathrm{MOF}$ (PCN-95) on human ovarian cancer cell lines (A2780) ${ }^{28}$ by in vitro cytotoxicity assays. Zirconium ( $\mathrm{Zr}$ ) is an established metal in bone implant materials, due to its mechanical strength and biocompatability with tissue promoting cell adhesion, growth and remodelling in osteoclasts and endothelial cells. It was anticipated that incorporation of $\mathrm{Zr}$ into the pyrene secondary building units (SBU) scaffolds would improve the physiochemical and biological properties relative to other common metals like Nickel or Iron, promoting bioactivity similar to $\mathrm{Zr}$ bioactive mesoporous scaffolds used in bone tissue reengineering. ${ }^{29}$ From these assays, the effective dose at fifty percent survival $\left(\mathrm{ED}_{50}\right)$ against A2780 (20 ppm) can be determined. The low dose in part due to uniform coating of the core, magnetically ordered surface crystallinity, nanoscale $(<20 \mathrm{~nm})$ size with ultrahigh surface area and shape for targeted drug delivery and cancer cell diagnostics. 
https://mc06.manuscriptcentral.com/cjc-pubs 


\section{Experimental}

All chemicals unless otherwise specified were All chemicals, solvents, and reagents were obtained from VWR International, (West Chester, PA) or Sigma-Aldrich (St. Louis, MO), The filtered ultrapure water was used (Ultraopure ${ }^{\mathrm{TM}}$, Barnstead, Dubuque, IA, referred to as distilled water), was used where water-based solvents were necessary. All solvents were reagent or high performance liquid chromatography (HPLC) grade. A facile solvothermal chemistry was employed to prepare PCN-95 motifs. The crystalline structure and crystallite size of NPs were investigated by Bruker D8 advance XRD using the copper $(\mathrm{Cu}) \mathrm{K}_{\alpha 1}$ line. The operating conditions were controlled at voltage of $40 \mathrm{kV}$ and current of $40 \mathrm{~mA}$. The scanning range varied from 10 to $100^{\circ}$ with scanning rate of $0.02 \%$. The transmission electron microscpy (FEI Company, Tecnai F20-G2 Hillsboro, OR) was operated at $200 \mathrm{kV}$ to study the morphology and crystallinity of the PCN-95 motifs in STEM, EDS and EELS mode. Human hTERT-RPE or A2780 cell cultures were maintained in a buffer consisting of essential medium with Earle's salts (Invitrogen/GIBCO Life Technologies, Inc., Carlsbad, CA) supplemented with 10\% heatinactivated fetal bovine serum, 100 units $/ \mathrm{ml}$ penicillin, $100 \mu \mathrm{g} / \mathrm{ml}$ streptomycin, $1.4 \mathrm{mM} \mathrm{L-}$ glutamine, maintained at $35^{\circ} \mathrm{C}$ in an incubator having an atmosphere of $95 \%$ air and $5 \% \mathrm{CO}_{2}$ and $75 \%$ humidity. The cells used were in log-phase growth. The cells were examined by microscopy and a random selection of a membrane integrity assay for lactate dehydrogenase (LDH). The appropriate kits were used following manufacturers protocols. Briefly, $50 \times 10^{3}-100 \times 10^{3}$ cells/wells were harvested from the culture plate. For the LDH, ROS or MTT assays, the appropriate probes were added to the cells in the dark with appropriate volumes $(5-100 \mu \mathrm{L} /$ well $)$ 
of buffers, reagents kits and standards without and with $5 \mu \mathrm{L}$ of PCN-95. The samples incubated for 45 minutes and fluorescence or absorbance measured.

\section{Results and Discussion}

To construct polymeric coordinated network (PCN)-95for cancer theranositics, pyrene linkers that exhibit strong fluorescence was selected as the functional group. Thus 4,4',4",4"'-(pyrene1,3,6,8-tetrayl)tetrabenzoic acid ( $\mathrm{H}_{4}$ TBAPy) containing pyrene as backbone was chosen as the

organic linker. ${ }^{30,31}$ Based on "hard and soft (Lewis) acids and bases" (HSAB) theory ${ }^{32}, \mathrm{Zr}^{4+}$ cation with high valence and closed shell electron configuration could form strong coordinative covalent bond with carboxylate and produce solid fluorescent frameworks. ${ }^{33-34}$ The solvothermal reactions between $\mathrm{H}_{4}$ TBAPy and $\mathrm{Zr}(\mathrm{IV})$ resulted light yellow polycrystal, abbreviated as PCN95 (Fig. 1).

$\mathbf{a}$

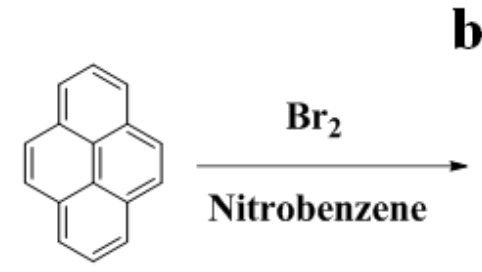

Pyrene<smiles>Brc1cc(Br)c2ccc3c(Br)cc(Br)c4ccc1c2c43</smiles><smiles>COC(=O)c1ccc(PO)cc1</smiles>

DME $\quad \operatorname{Pd}\left(\mathrm{PPh}_{3}\right)_{4} / \mathrm{CsF}$

d<smiles>O=C(O)c1ccc(-c2cc(-c3ccc(C(=O)O)cc3)c3ccc4c(-c5ccc(C(=O)O)cc5)cc(-c5ccc(C(=O)O)cc5)c5ccc2c3c54)cc1</smiles>
$\mathrm{H}_{4}$ TBAPy

MeOOC<smiles>C=CC(=C)c1ccc(F)cc1</smiles><smiles>COC(=O)c1ccccc1</smiles>

(i) $\mathrm{NaOH}$

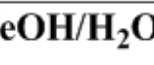
$\mathrm{MeOOC}$

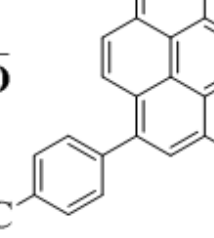<smiles>COC(=O)c1ccc(C)cc1</smiles> 
Fig. 1. The solvothermal chemistry was employed to produce PCN-95, $\boldsymbol{a}$ : the starting material used to synthesize ligand; $\boldsymbol{b}$ : bromination to expend the ligand size; $\boldsymbol{c}$ : the intermediate product to introduce tetra-topic ligands; $\boldsymbol{d}$ : the ligand with tetra-topic carboxylic groups, and $\boldsymbol{e}$ : synthesis of PCN-95 using solvothermal wet-chemistry.

The powder X-ray diffraction (PXRD, Fig. 2a) study indicated that PCN-95 displayed an isoreticular structure of PCN-222 (P6/mmm space group with a $=39.3875(7) \AA$ and $\mathrm{c}=$ 16.4829(3) $\AA, \alpha=90^{\circ}$ ). The secondary building unit (SBU) is $\mathrm{Zr}_{6}$-octahedron which has eight $\mu_{3}-\mathrm{OH}$ covering all triangular faces, eight normal $\mathrm{OH}$ and eight carboxylates (Fig. 2b). The dihedral angle between phenyl rings and pyrenes is 55.62 degree (Fig. 2c). Each $\mathrm{Zr}_{6} \mathrm{SBU}$ connects eight TBAPy linkers while each TBAPy linker connects to four $\mathrm{Zr}_{6}$ SBUs. Thus a three-dimensional (3D) framework is constructed with two types of one-dimensional (1D) channels running along c axis (Fig. 2c). The large hexagonal channel has $30 \AA$ diameters and the small triangular channel has $9 \AA$ diameters. This structure is identical to $\left[\mathrm{Zr}_{6}\left(\mu_{3}-\right.\right.$ $\left.\mathrm{OH})_{8}(\mathrm{OH})_{8}(\mathrm{TBAPy})_{2}\right](\mathrm{NU}-1000)$ reported by Hupp's group. ${ }^{38,39}$ Briefly, the PCN-95 were generated by a hydro-solvothermal approach to produce well-defined and highly crystallized coordinative compounds with a tetra-topic ligand as organic linker (Fig. 1d:). The nucleation of PCN-95 crystal units was promoted when the solvent molecules were evaporated from the solution. This rapid nucleation favoured the formation of PCN-95 along a defined-axis with controllable size and monodispersity. 


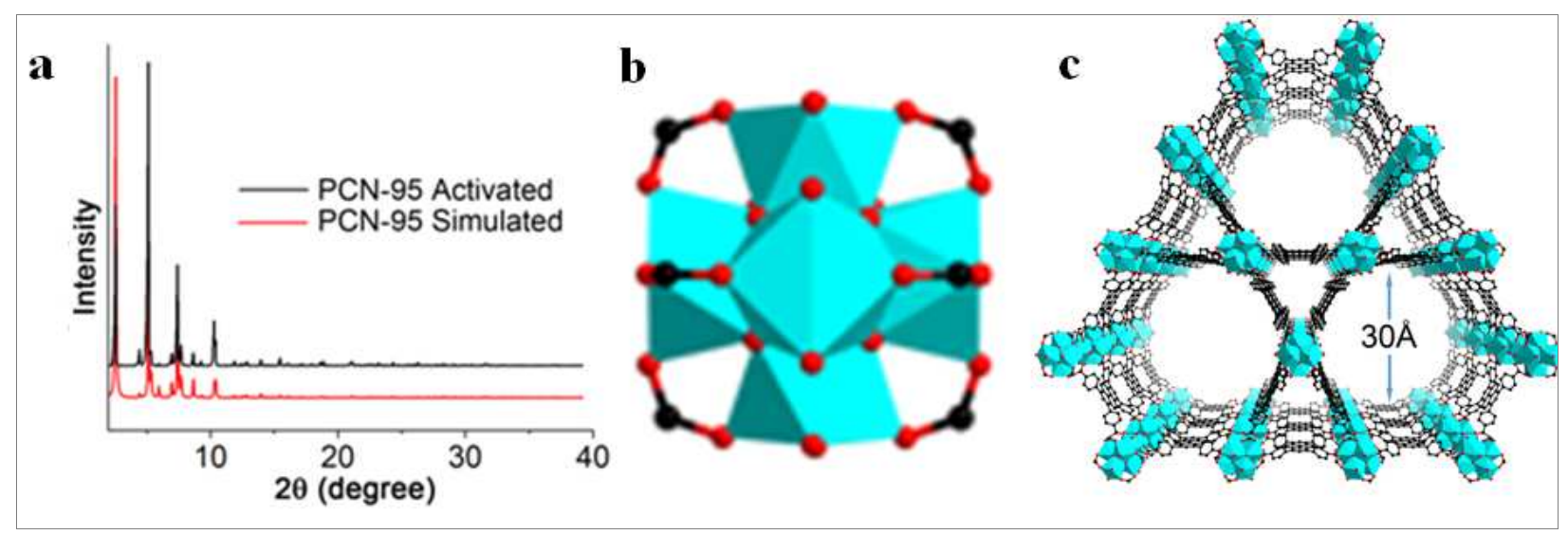

Fig. 2. The X-ray analysis of PCN-95 to determine its crystalline structure, $\boldsymbol{a}$ : PXRD patterns of PCN-95; $\boldsymbol{b}$ : 8-connected $\mathrm{Zr}_{6}$ clusters; $\boldsymbol{c}$ : crystal structure of PCN-95. All H atoms are omitted, for clarity. Atom color: black, C; red, O; blue polyhedron, Zr.

The transmission electron microscopy (TEM) images of PCN-95 (Fig. 3) indicate that the fabricated materials are rod-shaped crystals. ${ }^{35}$ Three different formulations of PCN-95 motifs were tested against the A2780 cell lines (Fig. 4) with the $\mathrm{ED}_{50}$ inhibition varying for each MOF and being approximately $10 \mu \mathrm{M}$ for PCN-95b (MOF-2). Higher doses $(>1 \mu \mathrm{M})$ were observed to be cytotoxic using the 3-(4,5-dimethylthiazol-2-yl)-2,5-diphenyltetrazolium bromide (MTT) assay. ${ }^{36}$ The TEM images show a control cell (left image) and a damage cell, in which extensive vascularisation is observed. From the figure, the cell appears to have a number of regions in which vesicle like formations are observed, tentatively indicating deoxyribonucleic acid (DNA) fragmentation and damage to the plasma membrane and eventual apoptosis, although the nanomaterials are not themselves cytotoxic upon use, as the time-course lactate dehydrogenase assay demonstrated in Fig 5, but exhibit plasma membrane disassembly under 150 minutes, resulting in partial lysis and internal macromolecule stress via reactive oxygen species (ROS, Fig 6) within the same time period for retinal pigment epithelium (RPE) cells. 
Coupled with $\mathrm{ED}_{50}$ data for $\mathrm{A} 2780$ cells, the mode of cellular inactivation appears to three-fold. Stress on the plasma membrane, denaturation of the DNA, internal macromolecules, such as respiratory proteins and general ROS stress to the mitochondria, resulting in apoptosis.
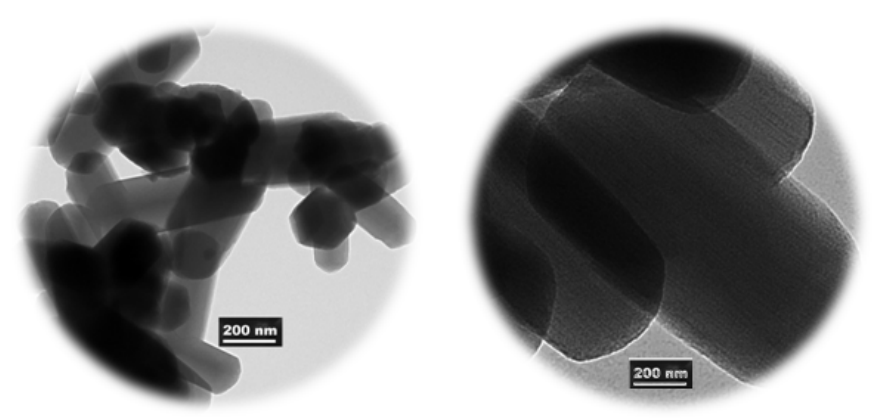

Fig. 3 The TEM analysis of PCN-95 to determine its morphology
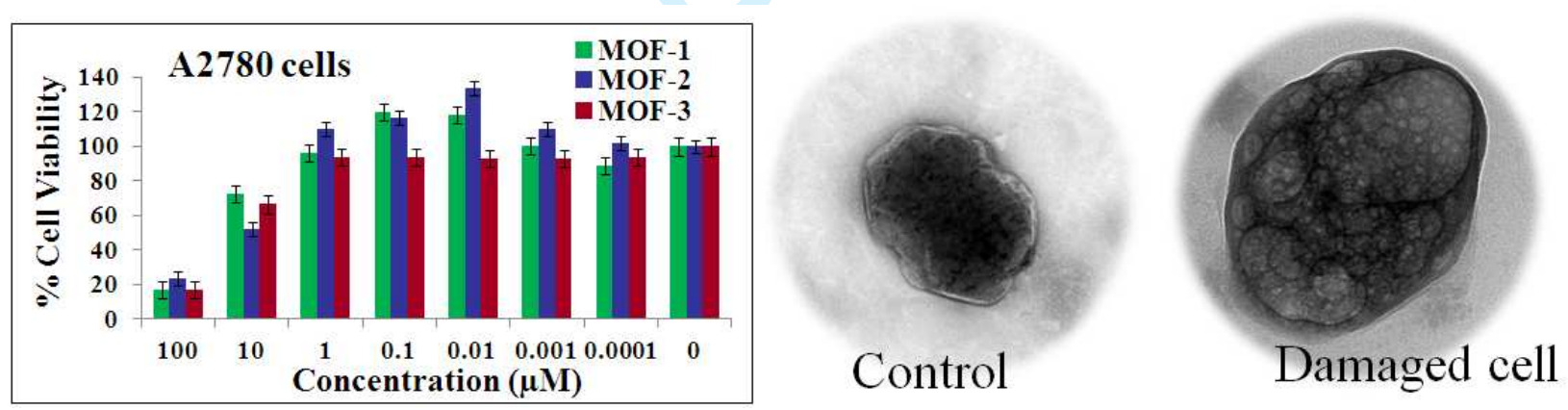

Fig. 4. Efficacy of PCN-95 motifs on Chinese hamster ovary (CHO) Cells, $\boldsymbol{a}$ : cell viability [Approximately $50 \times 10^{3}$ cells were used with varying MOF concentration. Samples ran in at least triplicate. Assay ran at room temperature. Error shown on graph]; $\boldsymbol{b}$ : the control of A2780; and $\boldsymbol{c}$ : damaged A2780 cell lines. 


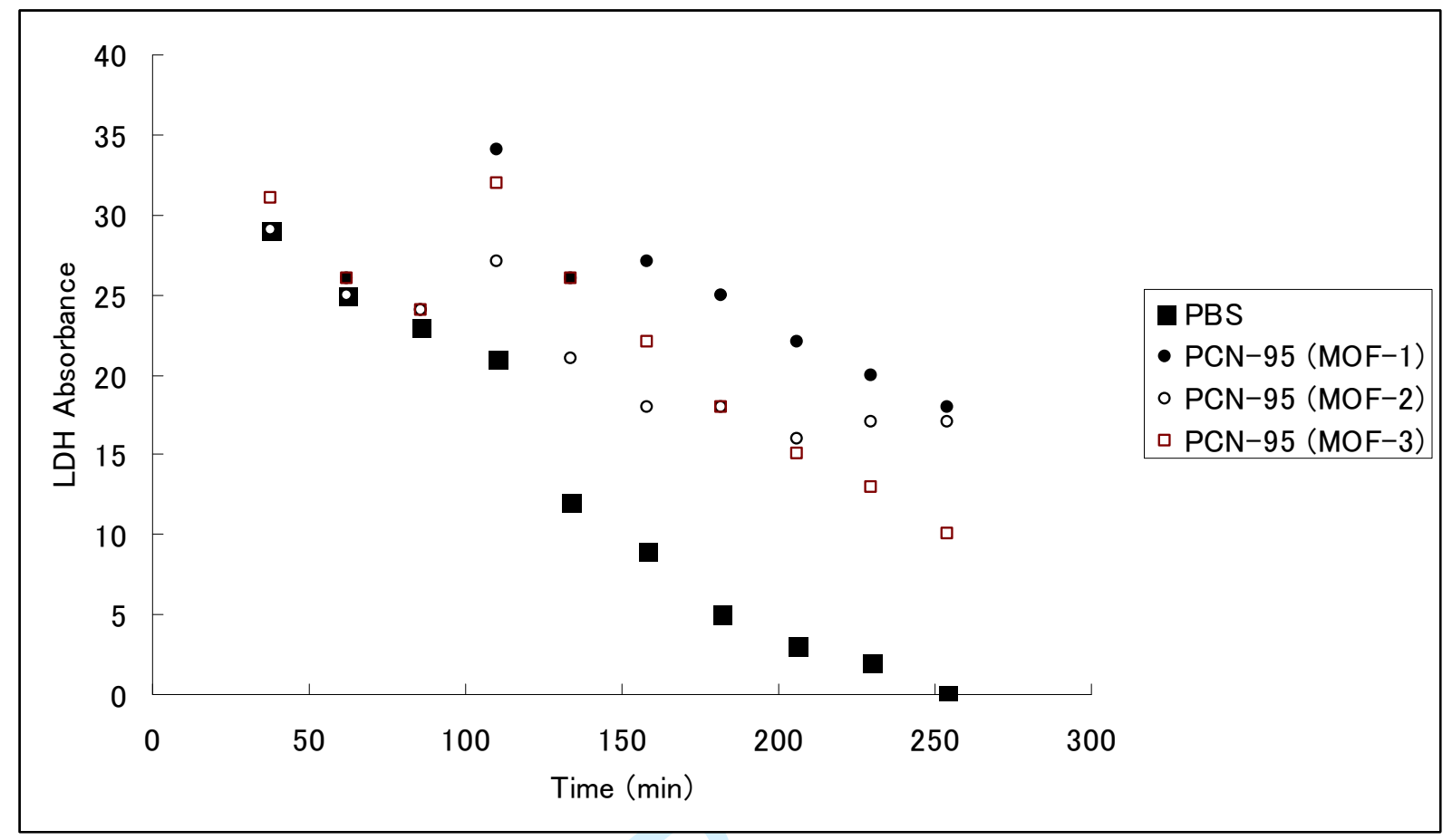

Fig. 5. Quantitatively measures lactate dehydrogenase (LDH) through the absorbance of formazan as a measure of cellular cytotoxicity of retinal pigment epithelium (RPE) whole cells by three formulations of PNC-95 MOFs compared to whole cells in phosphate buffered saline (PBS) control. [Approximately $120 \times 10^{3}$ cells/well were used, cells were incubated at $37^{\circ} \mathrm{C}$ in a constant humidity incubator, in which to each sample well 10 parts-per-million of MOF in dimethylsulfoxide (DMSO) was added to cells for 15 minutes followed by addition of LDH probe and incubation for 45 minutes followed by kinetic scan over 250 minutes at 30 minutes/scan. The absorbance's at $680 \mathrm{~nm}$ were subtracted from $490 \mathrm{~nm}$ and background adjusted from which LDH activity was determined]. The maximum error over the time period of 300 minutes was $<10 \%$. 


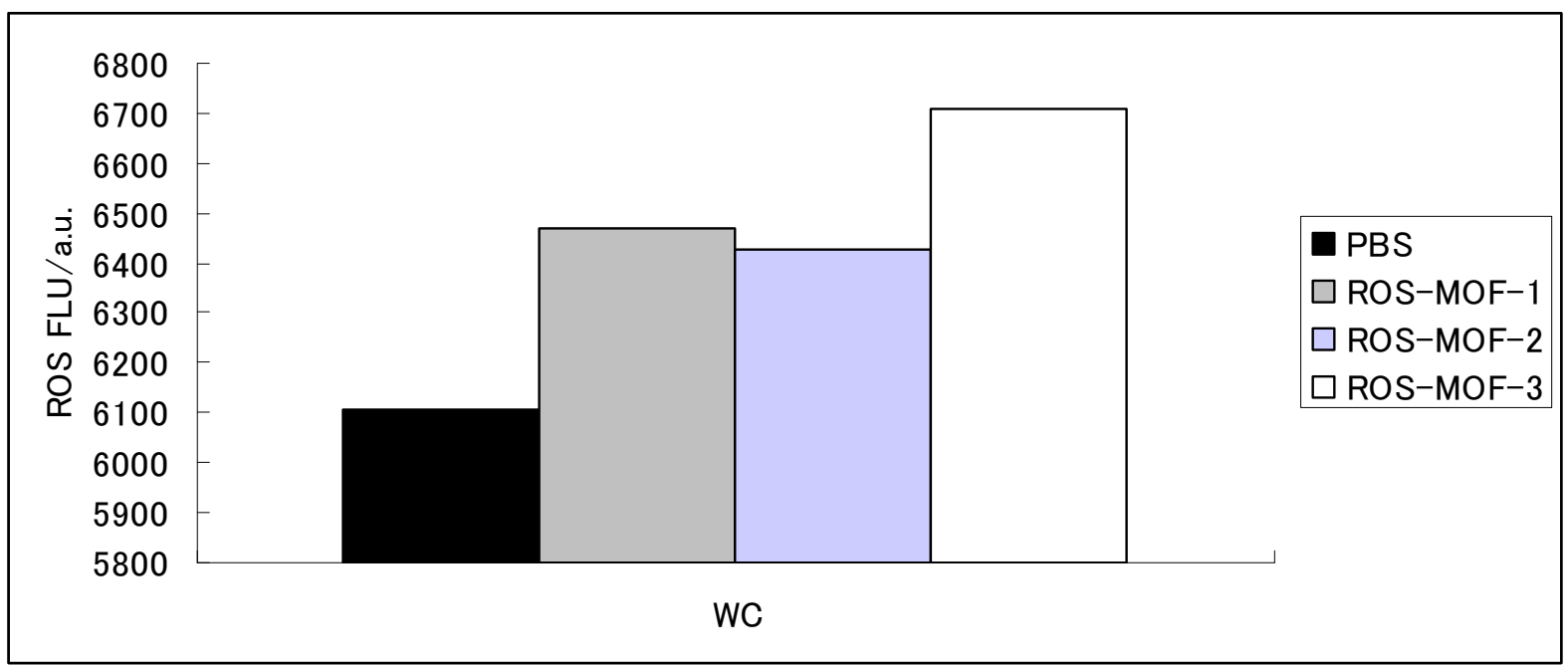

Fig. 6. Fluorescence of 5-(and-6)-chloromethyl-2',7'-dichlorodihydro-fluorescein diacetate, acetyl ester, a probe for reactive oxygen species (ROS) in retinal pigment epithelium (RPE) whole cells (WC) in phosphate buffered saline (PBS) used as control. [Approximately $120 \times 10^{3}$ cells/well were used, cells were incubated at $37^{\circ} \mathrm{C}$ in a constant humidity incubator. To each sample well 10 parts-per-million of the MOF agent in dimethylsulfoxide (DMSO) was added and allowed to incubate for 30 minutes followed by addition of ROS probe and further incubation for 30 minutes followed by measurement of ROS fluorescence at room temperature using a plate reader with Ex/Em: 492-495/517-527 nm filters]. The maximum error over the time period of 60 minutes was $<2 \%$ and values are significant at $95 \%$ confidence levels relative to controls. 
The mode of cell activation for PCN was further investigated by co-incubation of the MOF with specific agents whose mode of action was known (Fig 7). These agents were grouped into two broad class or classes with methylene blue as the standard singlet oxygen generator, or oxypurinol as an inhibitor of xanthine oxidase that is capable of synthesis in the cytoplasm or inhibitors of respiration such as oligomycin A, rotenone or cyanide. The kinetic profile was compared and contrasted to control (cells without addition of any factors) for approximately 3 hours. The profile of PCN alone and PCN with methylene blue are very similar for the first two hours and with oligomysin for the last hour (Fig 7a). Collectively these results indicate that PCN in part generates singlet oxygen that causes limited oxidative stress at the mitochondria, affecting cytochrome c oxidase and complex V. The use of oxypurinol was not designed to shut down nitric oxide (NO) synthesis, since NO was used as an assay, it was designed to slow down NO in the cytoplasm, leaving NO synthase (NOS) as the major enzyme in the cytoplasma and complex IV in the mitochondria as enzymes capable of synthesising NO. Co-addition of oxypurinol and PCN causes a slight decrease in NO production (sample C4) confirming that PCN does not act upon xanthine oxidase. Oligomycin A is a "leaky" inhibitor of complex IV on the $\mathrm{F}_{0}$ side, reducing adenosine triphosphate (ATP) production and reducing but not stopping electron flow down the electron transport chain. Rotenone on the other hand, inhibits complex I to ubiquinone electron transfer, reducing ATP production and stopping respiration, although electrons build up in the mitochondrial matrix that reduce molecular oxygen to radicals. Co-administration of suboptimal concentrations does reduce NO levels but not to the extent of methylene blue, suggesting that PCN acts further up the electron transport chain, but like rotenone contributes to the formation of radical oxygen species that cause oxidizes stress in the mitochondria, which in 
turn reduces mitochondrial and overall cellular NO. Lastly, sodium cyanide was used as reversible competitive inhibitor for Iron center of complex IV and stop electrons transport to complex IV. The drop in NO is more with cyanide than PCN alone and gets more pronounced at around $2.5 \mathrm{~h}$. To determine whether PCN is contributes to reduction of oxygen to oxygen, or generates singlet oxygen, other known standards were used (Fig 7b) alone and profiles compared. These standards could be grouped as oxidizers (hydrogen peroxide, sodium nitrite), reducers (citrate, dithranol) or inhibitors of respiration (FCCP, potassium ferrocyanide, $\mathrm{K}_{4}\left[\mathrm{Fe}(\mathrm{CN})_{6}\right] \bullet 3 \mathrm{H}_{2}$ coordinated with $\left[\mathrm{Fe}(\mathrm{CN})_{6}\right] 4^{-}$) or cell division (SN-38). Lastly, sodium nitroprusside was used as a slow NO release agent, to compare the capacity of PCN to store and release NO at a later time, when NO cellular levels drop. The results were compared with RPE whole cells (WC) without any supplements (sample A4) and the NO trends fall into two distinct trends. Considerably higher than NO levels across the $3 \mathrm{~h}$ were found for cells treated with nitrite anion, that is reduced to $\mathrm{NO}$ by the mitochondrial electron transport chain $\left[\mathrm{Fe}^{2+}+\mathrm{NO}_{2}^{-}+\mathrm{H}^{+} \rightarrow\right.$ $\left.\bullet \mathrm{NO}+\mathrm{OH}^{-}\right]$. Carbonyl cyanide-4-(trifluoromethoxy)phenylhydrazone (FCCP) uncouples the proton gradient in the electron transport chain, allowing protons to move across without ATP generation, which may be used by iron containing enzymes for generate additional NO from cellular nitrite. Hydrogen peroxide can also provide electrons to generate superoxide, which in turn can increase NO. Dithranol is a reducing agent and is known to act upon skin in promoting pro-inflammatory factors that control psoriasis through its oxidation and release of radicals. Sodium citrate is a mild reducing agent that can potentially be oxidized to carbon dioxide and water, increasing the acidity towards alkalinity. In a highly oxidizing environment, this process may lower the free radical content and lower oxidative stress in the mitochondria. The addition of citrate gave NO above controls that were constant across $3 \mathrm{~h}$ time period. Only other agent that 
gave a constant NO generation profile was SN-38, an anti-topoisomerase inhibitor, known to inhibit cell division. During the assay there is limited cell division countered by cell death induced by cell damage, or lack of nutrients. Addition of sup optional SN-38 reduced cell proliferation of RPE cells, which in turn would impact on total NO generation. The NO profile is lower than that observed with hydrogen peroxide but higher than citrate and constant across the 3h time period. Lastly, sodium nitroprusside $\left[\mathrm{Na}_{2}\left[\mathrm{Fe}(\mathrm{CN})_{5} \mathrm{NO}\right] \cdot 2 \mathrm{H}_{2} \mathrm{O}\right]$ was evaluated. It is a known NO releasing agent, although at suboptimal doses the amount of released NO was the least and similar to the effects of potassium ferrocyanide that slowly binds to the active site of complex IV, reducing NO levels. The PCN NO levels would correspond between the profile of citrate and SN-38, coupled with data from co-administration of inhibitors or methylene blue, it is suggestive PCN acts upon the mitochondria between complex III and IV and unlike cyanide or potassium ferrocyanide does not bind to complex IV, but facilitates the generation of singlet oxygen and NO generation, although the rate was relatively constant across three hours. 


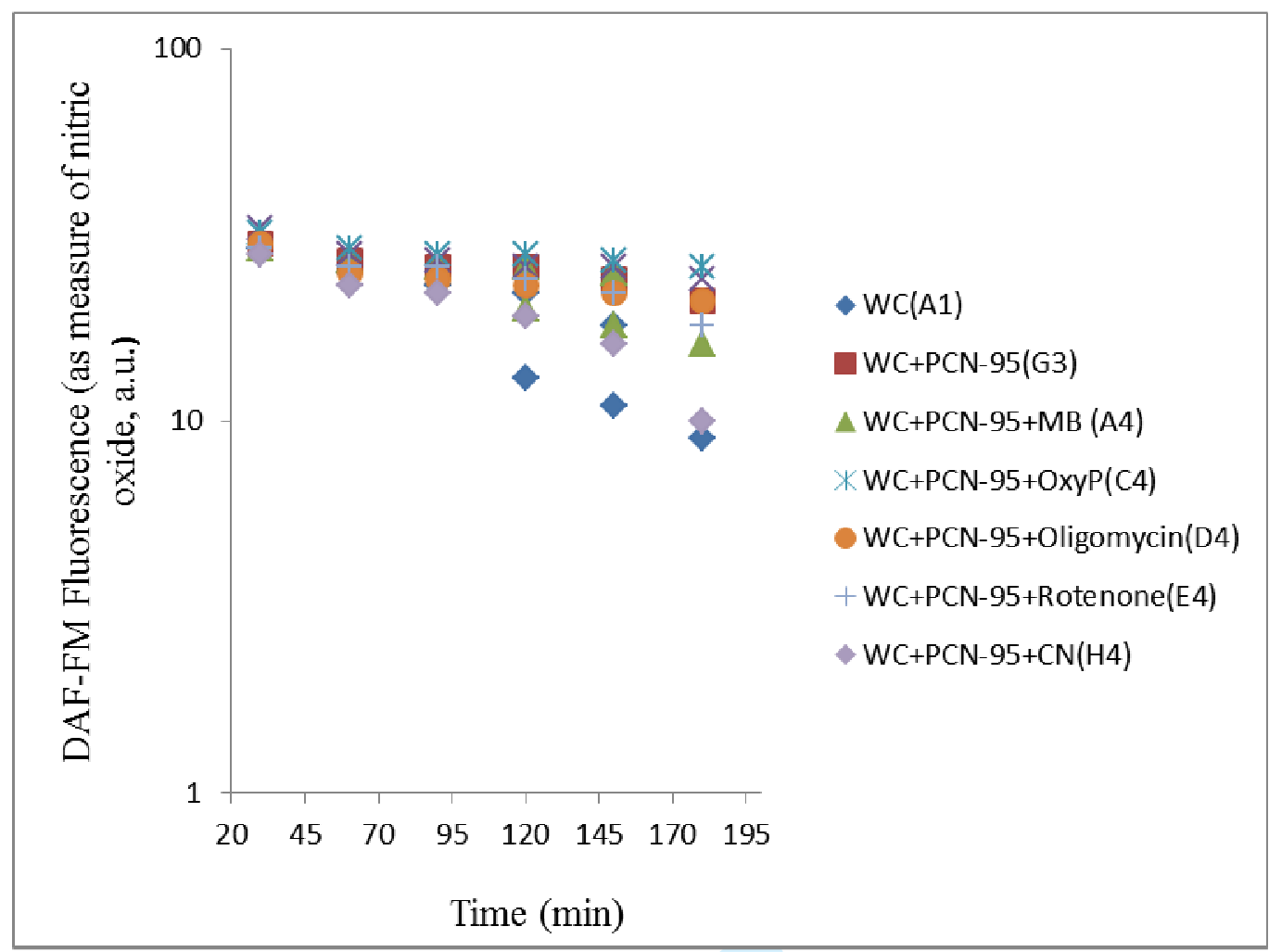

Fig. 7a. Log Fluorescence of 4-Amino-5-methylamino-2',7'-difluorofluorescein diacetate (DAFFM), a probe for nitric oxide (NO) in retinal pigment epithelium (RPE) whole cells (WC) in phosphate buffered saline (PBS as control) to either PCN alone or PCN in combination with other known inhibitors. [Approximately $120 \times 10^{3}$ cells/well in a total volume of $300 \mu \mathrm{L} / \mathrm{well}$ were used, cells were incubated with $25 \mu \mathrm{L}$ volume of 10 parts-per-million MOF in dimethylsulfoxide (DMSO) added to the well before cell addition. Other agents used were $25 \mu \mathrm{L}$ of $1 \%$ methylene blue in water (MB), $12.5 \mu \mathrm{L}$ of either $12.5 \mu \mathrm{M}$ of sodium cyanide $(\mathrm{CN})$ in water, $50 \mu \mathrm{M}$ oxypurinol in DMSO (OxyP), $10 \mu \mathrm{M}$ oligomycin in DMSO, or $10 \mu \mathrm{M}$ of Rotenone in DMSO to an equal volume of PCN. The samples were incubated at $37^{\circ} \mathrm{C}$ for 30 minutes in a constant humidity incubator and fluorescence read every 30 minutes over approximately 3 hours using a plate reader set to Ex/Em of DAF-FM: 495/515 nm excitation/emission wavelengths]. The largest percent error for any samples was less than $2.7 \%$. 


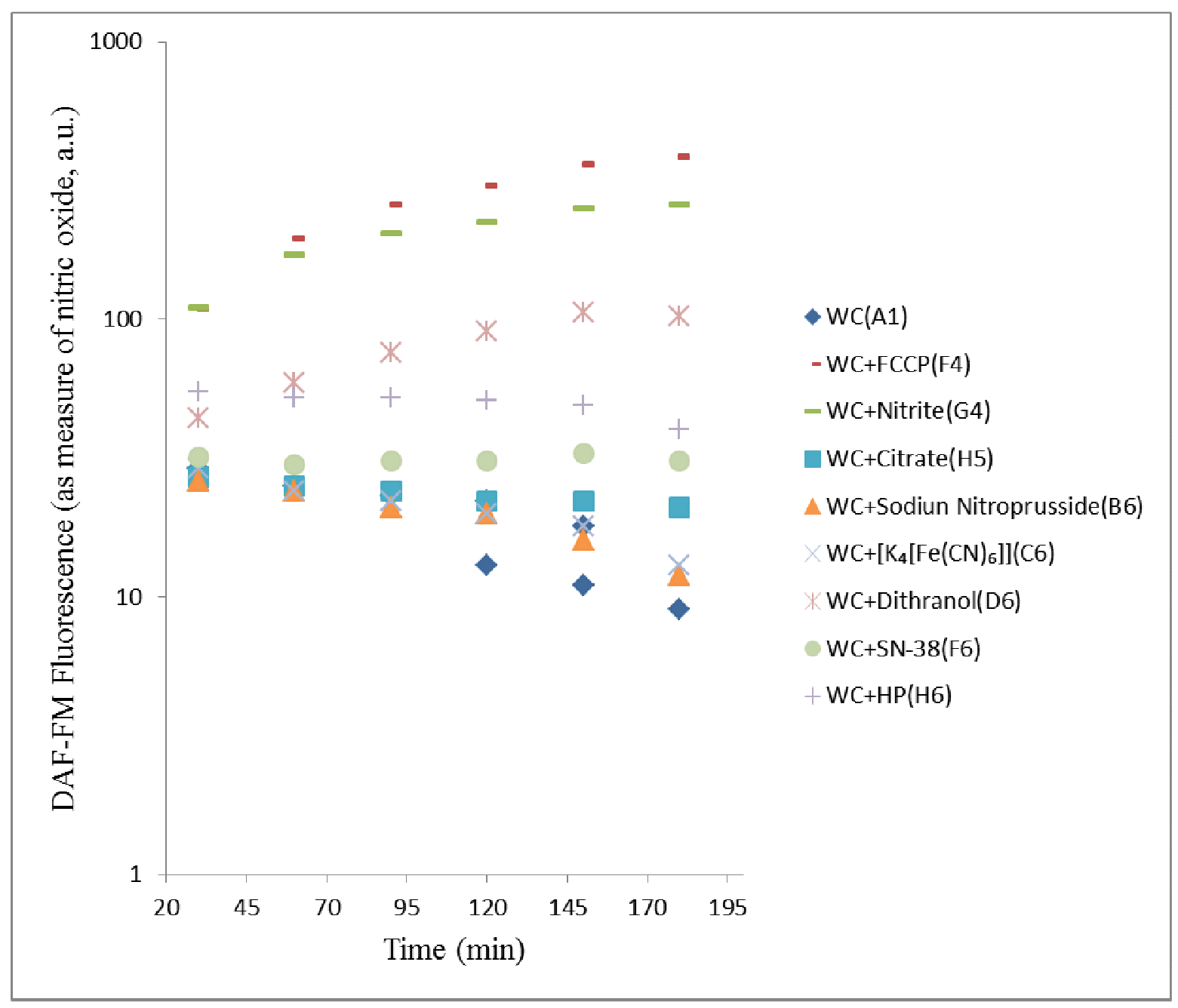

Fig. 7b. Log Fluorescence of 4-Amino-5-methylamino-2',7'-difluorofluorescein diacetate (DAFFM), a probe for nitric oxide (NO) in retinal pigment epithelium (RPE) whole cells (WC) in phosphate buffered saline (PBS as control) to other co-factors or inhibitors, servering as either negative or positive controls. [Approximately $120 \times 10^{3}$ cells/well in a total volume of 300 $\mu \mathrm{L} /$ well were used, cells were incubated with $25 \mu \mathrm{L}$ of the following agents in separate wells: 10 $\mu \mathrm{M}$ of Carbonyl cyanide-p-trifluoromethoxy-phenylhydrazone (FCCP) in DMSO, 1000 ppm of sodium nitrite in water, $500 \mathrm{ppm}$ of sodium citrate in water, $2 \mathrm{mM}$ of sodium nitroprusside in DMSO, $200 \mu \mathrm{M}$ of potassium ferrocyanide in DMSO, $1000 \mathrm{ppm}$ of dithranol in water, $10 \mathrm{ppm}$ of 
7-Ethyl-10-hydroxy-camptothecin (SN-38) in DMSO and 0.3\% hydrogen peroxide (HP) in water were. The control samples were incubated at $37^{\circ} \mathrm{C}$ for 30 minutes in a constant humidity incubator and fluorescence read every 30 minutes over approximately 3 hours using a plate reader set to Ex/Em of DAF-FM: 495/515 nm excitation/emission wavelengths]. The largest percent error for any control sample was less than $9.1 \%$, minimum of $1.2 \%$ and averaging at around 5\%.

Other possible modes of A2780 cell inhibition with PCN-95 involve incorporation of anti-cancer drugs, like SN-38 ${ }^{35}$ or through physical interactions such as magnetically induced hyperthermia 39 (Fig. 8a) through induction of localised heat resulting in the unzipping of deoxyribonucleic acid. Tagging of PCN with specific cellular determinants (CD) can target the MOF with embedded drugs to specific organs or cells such as kidney or lungs through specific receptor mediated pathways, which when in triggered on the pro-inflammatory side can facilitate apoptosis and cell death. Nitric oxide appears to be an important mediator that facilitates either cell protection at intermediate levels or cell death at low or high concentrations. PCN thus offers different modalities in its interaction with cells or organelles such as the mitochondria. With embedded SN-38 it can transport the anti-proliferation, with PCN alone, it can increase oxidative stress to partial generation of singlet oxygen, temporarily increase NO load on the cell and triggering cytochrome $\mathrm{c}$, similar to hydrogen peroxide induced toxicity, but not via binding to the active iron sites in complex IV like cyanide. 


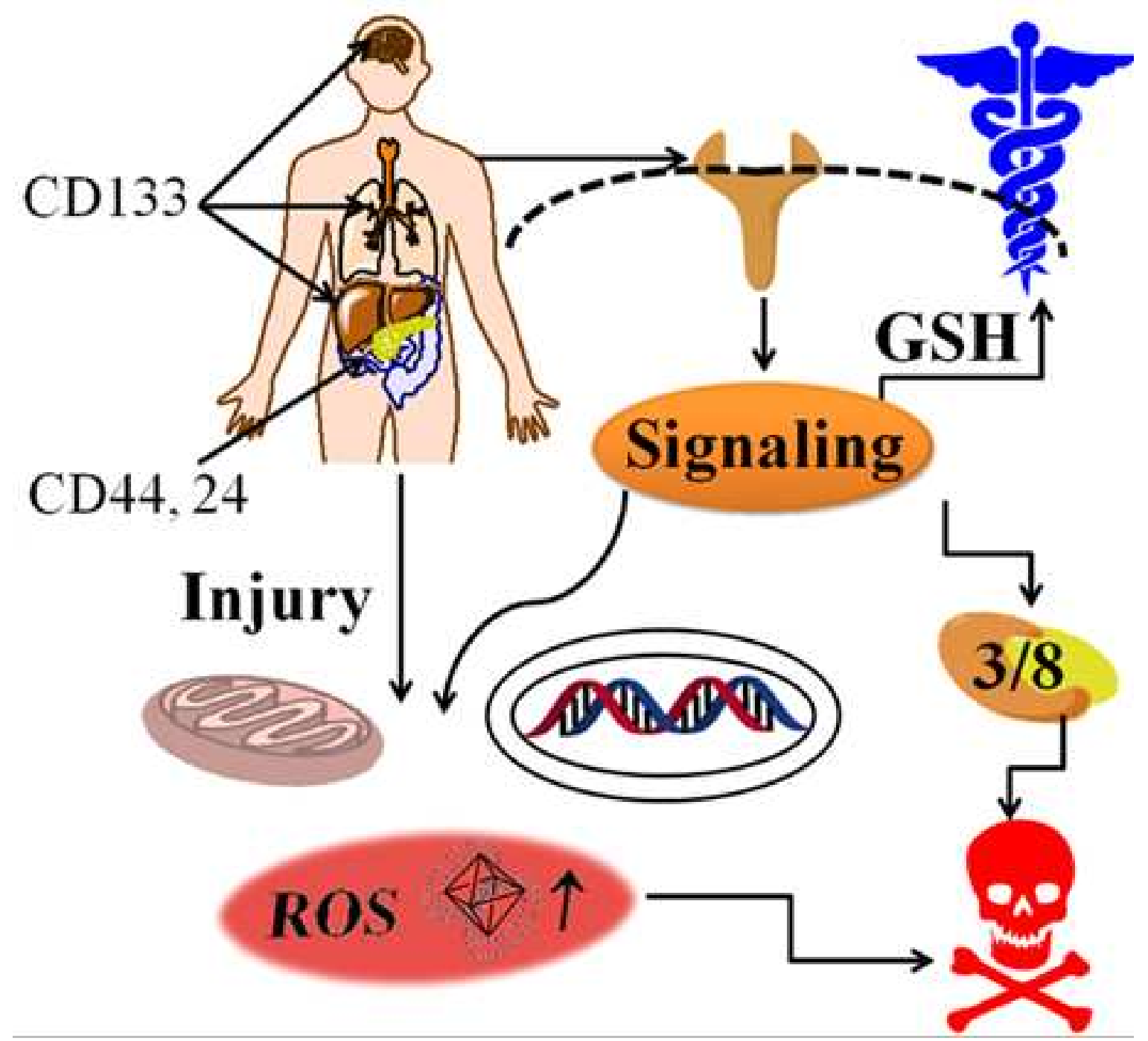

Fig. 8a. The hypothesized mechanism of cell apoptosis using PCN-95 as anti-cancer drug that has protective effect where cellular reducing equivalents dominant or pro-apoptotic when oxidative stress dominate, releasing cytochrome c, caspase 3 leading to DNA fragmentation and cell death. 


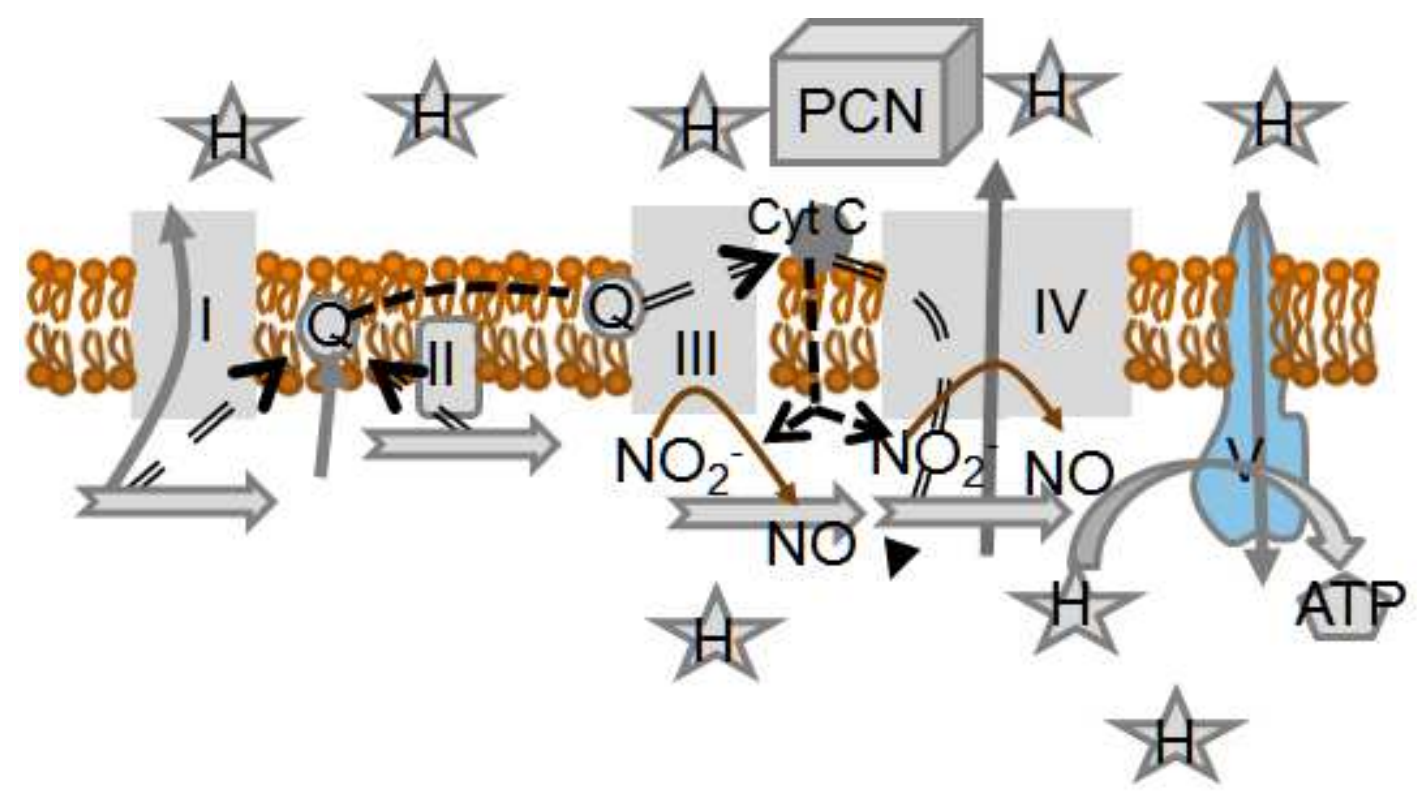

Fig. 8b. The hypothesized location of PCN95 on the mitochondria. Electron flow is indicated by the dotted arrows in black, proton flow by the solid gray arrows, substrate redox by the horizontal arrow ultimately generating water from molecular oxygen and ATP. Cellular nitrite can also be a "stored source" of available NO by reduction of nitrite to NO by cytochrome c.

\section{Conclusions}

In-vitro toxicity data indicated that $\mathrm{PCN}-95 \mathrm{IC}_{50}(20 \mathrm{ppb})$ is comparable to, or lower than the current first-line drug formulations. At the higher doses, the kinetics of action was found to be approximately ten-fold higher. These biological effects may be attributed to the unique n-topic TBAPy ligand bridging to a $\mu_{3}$ - centered cluster, in addition to high crystallinity and high aspect ratio. The latter factors lend themselves to high MOF-cell surface contact, which in turn leads to high degree of catalysis (e.g. depolarization of the mitochondrial membrane from the lactate dehydrogenase assay and inferred oxidation of critical proteins and DNA, using the ROS assay). Collectively, our results demonstrate a potentially viable approach in the treatment of solid tumours. PCN-95 shows high toxicity on cancer cell lines; via plasma membrane disassembly or 
ROS induced stress will provide a new avenue for MOFs applications in the biological realm.

\section{Acknowledgments}

The National Science Foundation (NSF-MRI, CBET 0821370), R. Welch Foundation (AC006), DOE (H-C Zhou) are duly acknowledged. The technical support from the Department of Chemistry at Texas A\&M University is also duly acknowledged. Professor Z. Luo is also duly acknowledged for his technical support on TEM collection. Dr. J. Wigle, 711 HPW/RHDO is also acknowledged for allowing access to his laboratory for Dr. Bashir to complete the RPE component.

\section{Author Contribution}

Drs. Zhou and Liu collectively conceived this project and oversaw the research progress. Four students, W. Zhang (Zhou), M. Maganti and B. Martinez (Bashir and J. Liu), and K. Vangara (Palakurthi) were trained to participate in sample preparation, biological assay, and toxicity against A2780. Dr. Palakurthi oversaw the biosafety aspects of the research and with Dr. Bashir, who contributed to the analyses of toxicity data and mechanism of RPE cells at RHDO. Dr. Lou collected the TEM images. Dr. Liu wrote the first draft and oversaw the submission process. Lastly, the second draft was edited by Dr. Bashir and the submission draft by all authors.

\section{References}

W. Zhuang, D. Yuan, J.R. Li, Z. Luo, H.-C. Zhou, S. Bashir, and J. Liu. Adv Healthcare Mater. $2012,1,225$.

2. R. Bardhan; S. Lal; A. Joshi; and N. J. Halas. Acc Chem Res. 2011, 44(10), 936.

3. W. T. Al-Jamal and K. Kostarelos. Acc Chem Res. 2011, 44(10), 1094. 
4. X. Li; Y. Qian; T. Liu; X..Hu; G. Zhang; Y. You and S. Liu. Biomaterials, 2011, 32(27), 6595.

5. J. E. Lee; N. Lee;. T. Kim; J. Kim and T. Hyeon. Acc Chem Res. 2011, 44(10) 893.

6. S. M. Janib; A. S. Moses and J. A. MacKay. Adv Drug Deliver Rev. 2010, 62(11) 1052.

7. A Mashal; B Sitharaman; X. Li; P. K. Avti; A. V Sahakian; J. H. Booske and S. C. Hagness.. Biomed Eng IEEE Transactions, 2010, 57(8) 1831.

8. K.Yang; L. Feng; X. Shi; and Z. Liu. (2013). Chem Soc Rev. 2013, 42(2) 530.

9. A.C. McKinlay; R.E. Morris; P. Horcajada; G. Férey; R. Gref; P. Couvreur and C. Serre. Angew. Chem. Int. Ed. Engl. 2010, 49(36) 6260.

10. S. L. James. Chem Soc Rev. 2003, 32(5) 276.

11. A. G Wong-Foy; A. J. Matzger and O. M Yaghi. J Am Chem Soc. 2006, 128(11) 3494.

12. H.-C. Zhou; J. R. Long and O. M. Yaghi. Chem Rev. 2012, 112(2) 673.

13. J. R. Long and O. M. Yaghi. Chem Soc Rev. 2009, 38(5) 1213.

14. J. L. Rowsell and O. M Yaghi. Micropor Mesopor Mat. 2004, 73(1) 3.

15. K. Koh; A. G. Wong-Foy and A. J. Matzger. Angew. Chem., Int. Ed. 2008, 47(4), 677.

16. L. .J. Murray; M. Dinca and J.R Long.Chem Soc Rev. 38(5) 1294.

17. A. R. Millward. \& O. M Yaghi. J Am Chem Soc. 127(51) 17998.

18. J. Li; R. R. J. Kuppler and H. C Zhou. Chem Soc Rev. 2009, 38(5) 1477

19. J. L. Rowsell and O. M Yaghi. Angew. Chem., Int. Ed. 44(30) 4670.

20. L. Ma; C Abney and W. Lin. Chem Soc Rev. 2009, 38(5) 1248.

21. J. Lee; O. K Farha; J Roberts; K. A Scheidt; S. T Nguyen and J. T Hupp. Chem Soc Rev. 2009, 38(5) 1450.

22. G. Lu and J. T Hupp. J. Am. Chem Soc. 2010, J Am Chem Soc. 132(23) 7832. 
23. L. E. Kreno; K. Leong; O. K. Farha; M Allendorf; R. P. Van Duyne and J. T. Hupp. Chem Rev. 2011, 112(2) 1105.

24. B. Chen; L. Wang; F. Zapata; G. Qian and E. B. Lobkovsky. J Am Chem Soc. 2008, 130(21) 6718.

25. P. Horcajada; C. Serre; G. Maurin; N. A. Ramsahye; F. Balas; E. M. Vallet-Reg and G. Férey. J Am Chem Soc. 2008, 130(21) 6774.

26. K. M Taylor-Pashow; J. D Rocca; X. Zie; S. Tran and W. Lin. J Am Chem Soc. 2009, 131(40) 14261.

27. J. Della Rocca; D. Liu and W. Lin. Acc Chem Res. 2011, 44(10) 957.

28. X. W. Hu; D. Meng and J. Fang. Carcinogenesis, 2008, 29(12) 2369.

29. Y. Zhu; Y. Zhang; C.Wu; Y. Fang; J. Yangand S. Wang. Micropor Mesopor Mat. 2011, 143(2) 311.

30. X. Shi; H. Wang; T. Han; X. Feng; B. Tong; J. Shi and Y. Dong. J Mater Chem. 2012, 22(36) 19296.

31. B. D. Wagner; G. J. McManus; B. Moulton and M. J. Zaworotko. Chem Comm. 2002, (18)

32. R. G. Pearson. J Chem Ed. 1987, 64(7), 561.

33. F. A. A. Paz; J. Klinowski; S. M. Vilela; E. J. P. Tom;. J. A. Cavaleiro and J. Rocha. Chem Soc Rev. 2012, 41(3) 1088.

34. H. Alawisi; B. Li; Y. He; H. D. Arman; A. M. Asiri; H. Wang and B. Chen. Cry Growth Des. 2014, 14(5) 2522.

35. K. K. Vangara; H. I. Ali; D. Lu; J. L. Liu; S Kolluru and S. Palakurthi. AAPS PharmSciTech. 2014, 15(2) 472.

36. M. V. Berridge; P. M. Herst and A. S. Tan. Biotech Annu Rev. 2005, 11127. 
37. P. Deria; J. E. Mondloch; E. Tylianakis; P. Ghosh; W. Bury; R. Q. Snurr and O. K. Farha. 2013, J Am Chem Soc. 2013, 135(45) 16801.

38. P. Deria; W. Bury; J. T. Hupp and O. K Farha. Chem Comm. 2014, 50(16) 1965.

39. G. P. Raaphorst; S. Doja; L. Davis; D. Stewart and C. E. Ng. Cancer Chemoth Pharm. 1996, 37(6) 574.

Tables, and figure captions.

Fig. 1. The solvothermal chemistry was employed to produce PCN-95, $\boldsymbol{a}$ : the starting material used to synthesize ligand; $\boldsymbol{b}$ : bromination to expend the ligand size; $\boldsymbol{c}$ : the intermediate product to introduce tetra-topic ligands; $\boldsymbol{d}$ : the ligand with tetra-topic carboxylic groups, and $\boldsymbol{e}$ : synthesis of PCN-95 using solvothermal wet-chemistry.

Fig. 2. The X-ray analysis of PCN-95 to determine its crystalline structure, $\boldsymbol{a}$ : PXRD patterns of PCN-95; $\boldsymbol{b}$ : 8-connected $\mathrm{Zr}_{6}$ clusters; $\boldsymbol{c}$ : crystal structure of PCN-95. All H atoms are omitted, for clarity. Atom color: black, C; red, O; blue polyhedron, Zr.

Fig. 3 The TEM analysis of PCN-95 to determine its morphology

Fig. 4. Efficacy of PCN-95 motifs on Chinese hamster ovary (CHO) Cells, $\boldsymbol{a}$ : cell viability [Approximately $50 \times 10^{3}$ cells were used with varying MOF concentration. Samples ran in at least triplicate. Assay ran at room temperature. Error shown on graph]; $\boldsymbol{b}$ : the control of A2780; and $\boldsymbol{c}$ : damaged A2780 cell lines.

Fig. 5. Quantitatively measures lactate dehydrogenase (LDH) through the absorbance of formazan as a measure of cellular cytotoxicity of retinal pigment epithelium (RPE) whole cells 
by three formulations of PNC-95 MOFs compared to whole cells in phosphate buffered saline (PBS) control. [Approximately $120 \times 10^{3}$ cells/well were used, cells were incubated at $37^{\circ} \mathrm{C}$ in a constant humidity incubator, in which to each sample well 10 parts-per-million of MOF in dimethylsulfoxide (DMSO) was added to cells for 15 minutes followed by addition of LDH probe and incubation for 45 minutes followed by kinetic scan over 250 minutes at 30 minutes/scan. The absorbance's at $680 \mathrm{~nm}$ were subtracted from $490 \mathrm{~nm}$ and background adjusted from which LDH activity was determined]. The maximum error over the time period of 300 minutes was $<10 \%$.

F ig. 6. Fluorescence of 5-(and-6)-chloromethyl-2',7'-dichlorodihydro-fluorescein diacetate, acetyl ester, a probe for reactive oxygen species (ROS) in retinal pigment epithelium (RPE) whole cells (WC) in phosphate buffered saline (PBS) used as control. [Approximately $120 \times 10^{3}$ cells/well were used, cells were incubated at $37^{\circ} \mathrm{C}$ in a constant humidity incubator. To each sample well 10 parts-per-million of the MOF agent in dimethylsulfoxide (DMSO) was added and allowed to incubate for 30 minutes followed by addition of ROS probe and further incubation for 30 minutes followed by measurement of ROS fluorescence at room temperature using a plate reader with Ex/Em: 492-495/517-527 nm filters]. The maximum error over the time period of 60 minutes was $<2 \%$ and values are significant at $95 \%$ confidence levels relative to controls. Fig. 7a. Log Fluorescence of 4-Amino-5-methylamino-2',7'-difluorofluorescein diacetate (DAFFM), a probe for nitric oxide (NO) in retinal pigment epithelium (RPE) whole cells (WC) in phosphate buffered saline (PBS as control) to either PCN alone or PCN in combination with other known inhibitors. [Approximately $120 \times 10^{3}$ cells/well in a total volume of $300 \mu \mathrm{L} / \mathrm{well}$ were used, cells were incubated with $25 \mu \mathrm{L}$ volume of 10 parts-per-million MOF in dimethylsulfoxide (DMSO) added to the well before cell addition. Other agents used were $25 \mu \mathrm{L}$ 
of $1 \%$ methylene blue in water (MB), $12.5 \mu \mathrm{L}$ of either $12.5 \mu \mathrm{M}$ of sodium cyanide $(\mathrm{CN})$ in water, $50 \mu \mathrm{M}$ oxypurinol in DMSO (OxyP), $10 \mu \mathrm{M}$ oligomycin in DMSO, or $10 \mu \mathrm{M}$ of Rotenone in DMSO to an equal volume of PCN. The samples were incubated at $37^{\circ} \mathrm{C}$ for 30 minutes in a constant humidity incubator and fluorescence read every 30 minutes over approximately 3 hours using a plate reader set to Ex/Em of DAF-FM: 495/515 nm excitation/emission wavelengths]. The largest percent error for any samples was less than $2.7 \%$.

Fig. 7b. Log Fluorescence of 4-Amino-5-methylamino-2',7'-difluorofluorescein diacetate (DAFFM), a probe for nitric oxide (NO) in retinal pigment epithelium (RPE) whole cells (WC) in phosphate buffered saline (PBS as control) to other co-factors or inhibitors, servering as either negative or positive controls. [Approximately $120 \times 10^{3}$ cells/well in a total volume of 300 $\mu \mathrm{L} /$ well were used, cells were incubated with $25 \mu \mathrm{L}$ of the following agents in separate wells: 10 $\mu \mathrm{M}$ of Carbonyl cyanide-p-trifluoromethoxy-phenylhydrazone (FCCP) in DMSO, 1000 ppm of sodium nitrite in water, $500 \mathrm{ppm}$ of sodium citrate in water, $2 \mathrm{mM}$ of sodium nitroprusside in DMSO, $200 \mu \mathrm{M}$ of potassium ferrocyanide in DMSO, $1000 \mathrm{ppm}$ of dithranol in water, $10 \mathrm{ppm}$ of 7-Ethyl-10-hydroxy-camptothecin (SN-38) in DMSO and 0.3\% hydrogen peroxide (HP) in water were. The control samples were incubated at $37^{\circ} \mathrm{C}$ for 30 minutes in a constant humidity incubator and fluorescence read every 30 minutes over approximately 3 hours using a plate reader set to Ex/Em of DAF-FM: 495/515 nm excitation/emission wavelengths]. The largest percent error for any control sample was less than $9.1 \%$, minimum of $1.2 \%$ and averaging at around $5 \%$.

Fig. 8a. The hypothesized mechanism of cell apoptosis using PCN-95 as anti-cancer drug that has protective effect where cellular reducing equivalents dominant or pro-apoptotic when 
oxidative stress dominate, releasing cytochrome c, caspase 3 leading to DNA fragmentation and cell death.

Fig. 8b. The hypothesized location of PCN95 on the mitochondria. Electron flow is indicated by the dotted arrows in black, proton flow by the solid gray arrows, substrate redox by the horizontal arrow ultimately generating water from molecular oxygen and ATP. Cellular nitrite can also be a "stored source" of available NO by reduction of nitrite to NO by cytochrome c. 